\title{
ADVANCES IN PS MANIPULATIONS AND LASER STUDIES IN THE AEgIS EXPERIMENT*
}

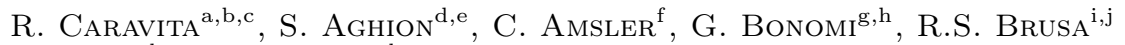
M. Caccia ${ }^{\mathrm{e}, \mathrm{k}}$, F. Castelli ${ }^{\mathrm{e}, \mathrm{l}}$, G. Cerchiari $^{\mathrm{m}}$, D. Comparat $^{\mathrm{n}}$, G. Consolati $^{\mathrm{d}, \mathrm{e}}$ A. Demetrio ${ }^{\circ}$, L. Di Noto ${ }^{\mathrm{b}, \mathrm{c}}$, M. Doser ${ }^{\mathrm{a}}$, C. Evans $^{\mathrm{d}, \mathrm{e}}$, R. Ferragut $^{\mathrm{d}, \mathrm{e}}$

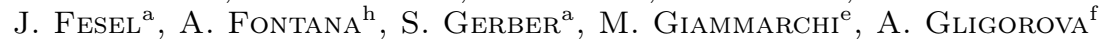

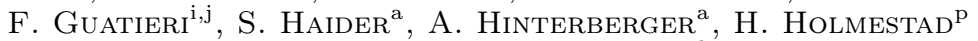

A. Kellerbauer ${ }^{\mathrm{m}}$, O. Khalidova ${ }^{\mathrm{a}}$, D. KrasnickÝ ${ }^{\mathrm{b}, \mathrm{c}}, \mathrm{K}$. Lagomarsino $^{\mathrm{b}, \mathrm{c}}$ P. Lansonneur ${ }^{q}$, P. Lebrun ${ }^{q}$, C. Malbrunot ${ }^{a}, f$, S. Mariazzi $^{r}$, J. Marton $^{f}$ V. Matveev ${ }^{\mathrm{s}, \mathrm{t}}$, Z. Mazzotta ${ }^{\mathrm{e}, \mathrm{n}}$, S.R. MÜller ${ }^{\mathrm{o}}$, G. Nebbia $^{\mathrm{r}}$, P. Nedelec ${ }^{\mathrm{q}}$

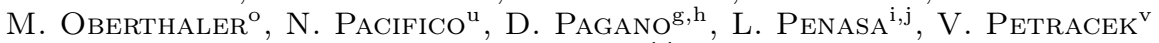
F. Prelz ${ }^{\mathrm{e}}$, M. Prevedelli ${ }^{\mathrm{w}}$, L. Ravelli ${ }^{\mathrm{i}, \mathrm{j}}$, B. RienäCker ${ }^{\mathrm{a}}$, J. RoberT ${ }^{\mathrm{n}}$

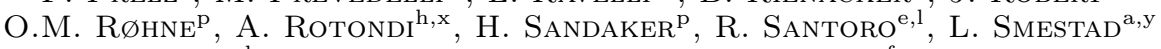

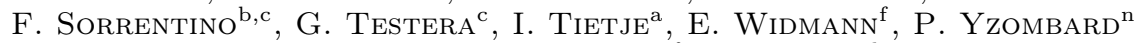

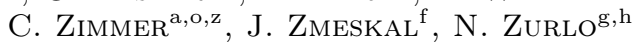

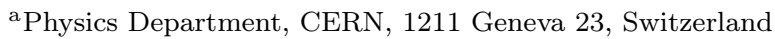

${ }^{b}$ Department of Physics, University of Genova, via Dodecaneso 33, 16146 Genova, Italy

${ }^{\mathrm{c} I N F N}$ Genova, via Dodecaneso 33, 16146 Genova, Italy

deolitecnico of Milano, Piazza Leonardo da Vinci 32, 20133 Milano, Italy e INFN sezione di Milano, via Celoria 16, 20133 Milano, Italy

${ }^{\mathrm{f}}$ Stefan Meyer Institute for Subatomic Physics, Austrian Academy of Sciences Boltzmanngasse 3, 1090 Vienna, Austria

g Department of Mechanical and Industrial Engineering, University of Brescia via Branze 38, 25123 Brescia, Italy

h INFN Pavia, via Bassi 6, 27100 Pavia, Italy

${ }^{\mathrm{i}}$ Department of Physics, University of Trento, via Sommarive 14, 38123 Povo, Trento, Italy ${ }^{j}$ TIFPA/INFN Trento, via Sommarive 14, 38123 Povo, Trento, Italy

${ }^{\mathrm{k}}$ Department of Science, University of Insubria, Via Valleggio 11, 22100 Como, Italy

${ }^{1}$ Department of Physics, Università degli studi di Milano, via Celoria 16, 20133 Milano, Italy

${ }^{\mathrm{m}}$ Max Planck Institute for Nuclear Physics, Saupfercheckweg 1, 69117 Heidelberg, Germany

${ }^{\mathrm{n}}$ Lab. Aimé Cotton, CNRS, Univ. Paris-Sud, ENS Cachan, Bâtiment 505, 91405 Orsay, France ${ }^{\circ}$ Kirchhoff-Institute for Physics, Heidelberg University Im Neuenheimer Feld 227, 69120 Heidelberg, Germany

${ }^{\mathrm{P}}$ Department of Physics, University of Oslo, Semælands vei 24, 0371 Oslo, Norway

${ }^{q}$ Institute of Nuclear Physics, CNRS/IN2p3, University of Lyon 1, 69622 Villeurbanne, France ${ }^{\mathrm{r}}$ INFN Padova, via Marzolo 8, 35131 Padova, Italy

s Joint Institute for Nuclear Research, Dubna, Russia

${ }^{\mathrm{t}}$ Institute for Nuclear Research of the Russian Academy of Science, Moscow, Russia

unstitute of Physics and Technology, University of Bergen, Alleegaten 55, 5007 Bergen, Norway

${ }^{\vee}$ Czech Technical University, Prague, Bøehová 7, 11519 Prague 1, Czech Republic

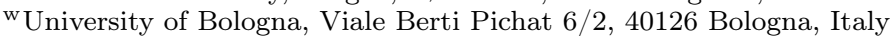

${ }^{\mathrm{x}}$ Department of Physics, University of Pavia, via Bassi 6, 27100 Pavia, Italy

y The Research Council of Norway, P.O. Box 564, 1327 Lysaker, Norway

${ }^{z}$ Dept. of Physics, Heidelberg University, Im Neuenheimer Feld 226, 69120 Heidelberg, Germany

(Received September 21, 2017)

* Presented at the $2^{\text {nd }}$ Jagiellonian Symposium on Fundamental and Applied Subatomic Physics, Kraków, Poland, June 3-11, 2017. 
Positronium (Ps), the unstable bound state of electron and positron, is a valuable system for neutral antimatter spectroscopic studies and for antihydrogen production. Forming a pulsed beam cold antihydrogen using charge-exchange with the Rydberg Ps is the goal of the AEgIS Collaboration, which aims to measure gravity on neutral antimatter. Recent results achieved in producing, manipulating and studying Ps are summarized. Ps has been first produced with mesoporous silica targets in a reflection geometry. Spectroscopy of Ps $n=3$ state has been conducted, yielding as a byproduct an independent estimate of the produced Ps temperature. Efficient laser excitation to the Rydberg levels was then achieved, validating the proof-of-concept of AEgIS. Subsequently, production of Ps from a new class of transmission targets was also achieved, opening the possibility for future experiments.

DOI:10.5506/APhysPolB.48.1583

\section{Introduction}

Measuring directly and accurately gravity on a pulsed beam of cold antihydrogen is the ambitious goal of the AEgIS Collaboration at CERN Antiproton Decelerator [1]. Its measurement scheme is conceptually straightforward: observe the free-fall parabolic trajectory of a cloud of antihydrogen atoms in a constant (Earth's) gravitational field. By measuring the time of flight of the falling atoms $\tau$ and their vertical displacement $\Delta y$ using a moiré deflectometer (the classical counterpart of the Talbot-Lau interferometer), $g$ can be promptly worked out from $\Delta y=g \tau^{2}$ (see [2]).

The scheme chosen by AEgIS to produce a pulsed source of antihydrogen atoms is based on a double charge-exchange reaction between cold trapped antiprotons and Rydberg-excited positronium (Ps) atoms, first demonstrated experimentally by the ATRAP Collaboration [3]

$$
\bar{p}^{-}+\mathrm{Ps}^{*} \longrightarrow \bar{H}^{*}+e^{-},
$$

where wildcard * indicates atoms in the Rydberg states. This formation process is an alternative to the traditional three-body recombination scheme (see [4]) adopted by the other experiments in the AD. Thanks to its much higher production cross-section scaling as the fourth power of the principal quantum number of Ps [5], antihydrogen can be formed in-flight (i.e. with the knowledge of the formation instant) by sending a bunch of neutral Ps* over trapped antiprotons. Antiprotons are left unperturbed in the trap during this process, potentially overcoming a limitation of three-body recombination schemes where antiproton/positron plasma mixing and trap potential movements are sources of plasma heating affecting the produced antihydrogen temperature. 
A sketched scheme of the experiment is shown in Fig. 1, left panel. Figure 1, right panel, shows instead a picture of the actual setup at CERN currently in data taking. A Ps converter made of mesoporous silica is placed about $1.7 \mathrm{~cm}$ facing downwards towards the plasma of trapped antiprotons held in a Malmberg-Penning trap. The electrodes of this trap have a custom design with an entrance grid on the upper side to let Ps* atoms enter the trap. Ps is produced from positrons implanted with keV energy inside the converter, where ground state Ps atoms are produced with high efficiency (both in the para- and orto-states, with lifetimes of $0.125 \mathrm{~ns}$ and $142 \mathrm{~ns}$ respectively). Para-Ps atoms annihilate immediately, while ortho-Ps atoms diffuse in the nanochannels and loose energy by collisions on the walls. They finally are re-emitted into vacuum with an overall efficiency of about $35 \%$ $($ see $[6,7])$.
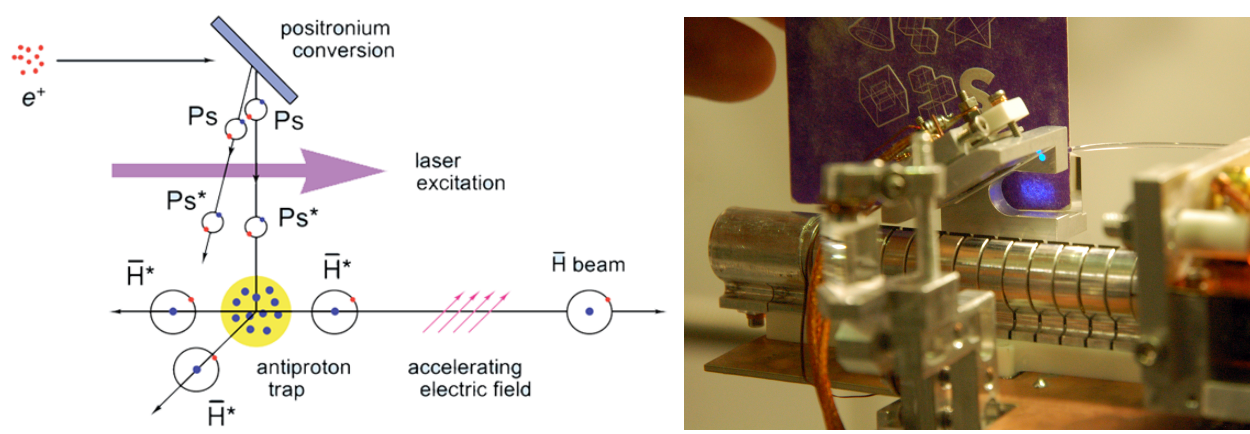

Fig. 1. Left panel: schematic drawing of AEgIS experimental layout to produce antihydrogen by charge-exchange with the Rydberg-excited positronium. Right panel: a view of the Malmberg-Penning traps used to capture antiprotons.

Two pulses of laser radiation are then sent synchronously in front of the converter (from the side) to excite the emitted fraction of ortho-Ps to Rydberg levels, using a two-step excitation scheme $1 \rightarrow 3 \rightarrow 15-23$ (see [8]). The total lifetime of $\mathrm{Ps}^{*}$ atoms is much higher than ortho-Ps (tens of $\mu \mathrm{s}$ to ms, depending on the substrate), allowing the atoms to fly towards the antiproton storage trap and perform charge-exchange with the antiprotons waiting inside.

\section{Experimental setup for Ps studies}

A simplified setup for dedicated Ps studies, directly connected to AEgIS positron system, has been envisaged by the collaboration to demonstrate its proof-of-principle in-flight laser excitation of Ps, and for further research and development. The setup is composed of an ultra-high vacuum multi- 
viewport test chamber, a mixed magnetic/electrostatic transfer line and the AEgIS positron system (sketched in Fig. 2), as well as AEgIS laser system (installed at some distance).

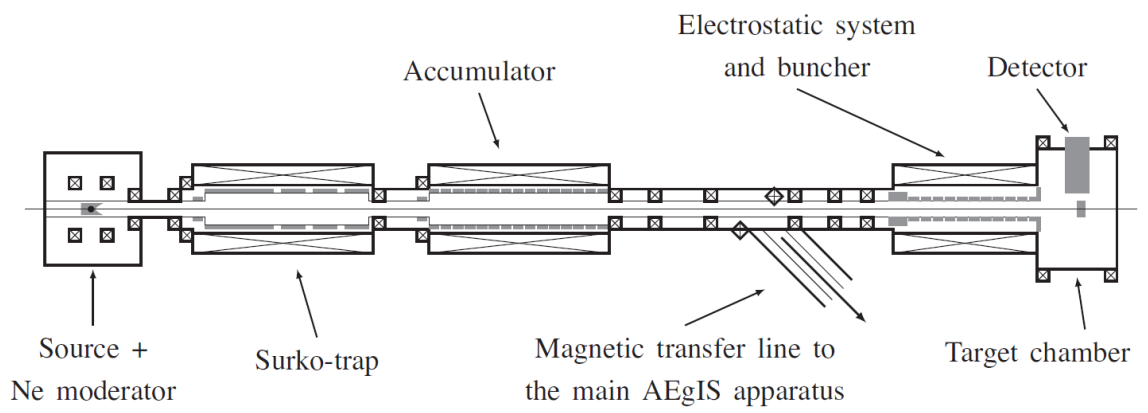

Fig. 2. Sketch of the AEgIS positron system.

Positrons are first emitted from a $9 \mathrm{mCi}{ }^{22} \mathrm{Na} \beta^{+}$source. Then they get slowed down to kinetic energies of a few $\mathrm{eV}$ in a solid Ne moderator [9] to be trapped in a so-called Surko-trap using buffer gas cooling [10]. Subsequently, positrons are transferred to a cylindrical Penning trap in a $0.1 \mathrm{~T}$ magnetic field where several pulses from the trap are accumulated. A rotating-wall drive [11] is applied on the central electrodes of the trap, radially compressing the plasma to $\sim \mathrm{mm}$ and increasing the $e^{+}$lifetime in the trap by avoiding radial losses on the electrode walls. Compressed $e^{+}$plasma are then extracted by fast-switching the electric potential on the electrodes. Positrons leave the accumulator in form of $20 \mathrm{~ns}$ bunches of $\sim 10^{7}$ positrons at $100 \mathrm{eV}$ axial energy. The cloud is transported to a magnetic-free region where it gets further accelerated and compressed in time using a 24-electrode buncher [7]. Positrons are implanted in the Ps conversion target (installed on a movable actuator in the center of the test chamber, see Fig. 3) with a final kinetic energy of $3.3 \mathrm{keV}$ and a time spread is of 7-9 ns FWHM.

A microchannel plate detector (MCP) in the center of the chamber is used to optimize the transport and to characterize the spot dimension of positrons impinging on the target. Usually, 30-40\% of positrons released from the accumulator hit the sample in a spot with a full-width at tenth maximum of $\leq 4 \mathrm{~mm}$. Two symmetric coils generating a magnetic field perpendicular to the sample are used to increase the positron transport efficiency onto the target. The target is kept at room temperature in a $25 \mathrm{mT}$ magnetic field environment and in presence of a $300 \mathrm{~V} \mathrm{~cm}^{-1}$ on average, mostly parallel to the magnetic field in the laser excitation region, produced by the last electrode of the buncher that acts as an electrostatic lens. 


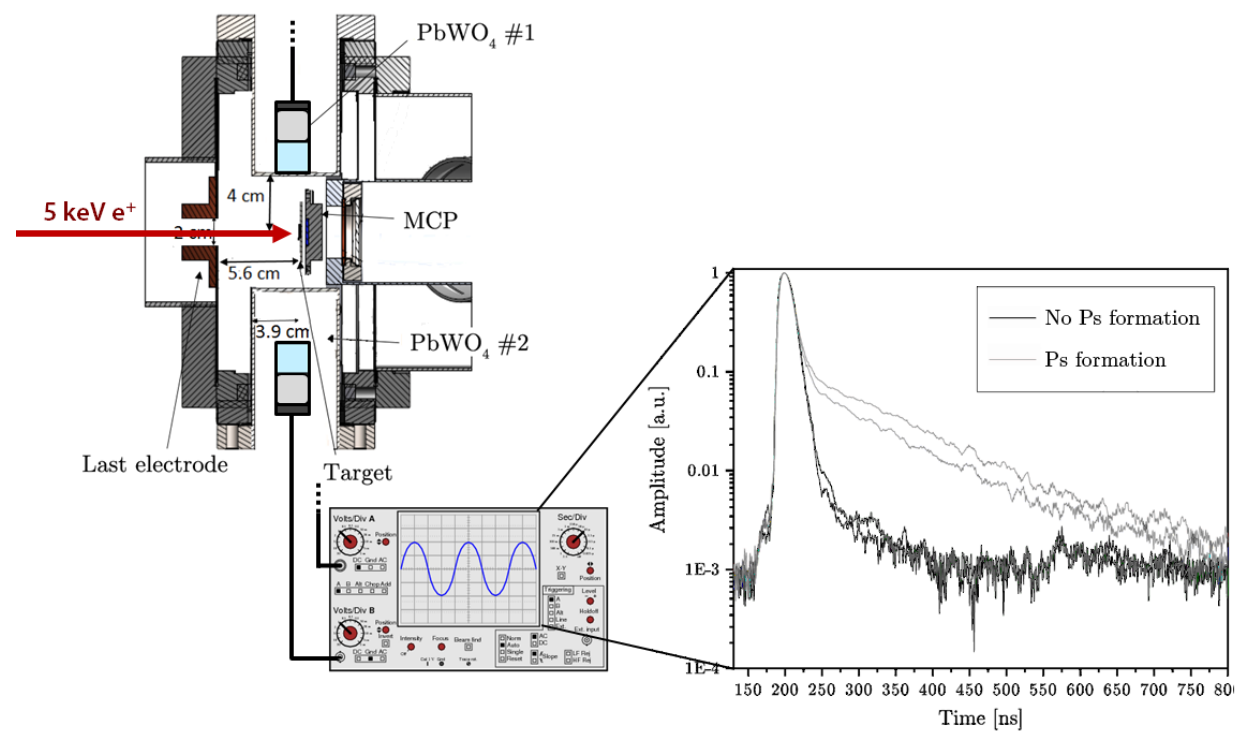

Fig. 3. (Colour on-line) Sketch of AEgIS dedicated setup for Ps spectroscopy experiments.

Ps formation is monitored using single-shot positron annihilation lifetime spectroscopy (SSPALS) [12, 13]. Briefly, a $20 \times 25 \times 25 \mathrm{~mm}$ lead tungstate $\left(\mathrm{PbWO}_{4}\right)$ scintillator [13] coupled to a Hamamatsu R11265-100 photomultiplier tube (PMT) placed $40 \mathrm{~mm}$ above the sample records the $\gamma$ rays emitted by Ps annihilations. A $1 \mathrm{GHz} 12$-bit scope digitizes the detector signal, which typically presents a sharp annihilation peak due to direct $e^{+}$annihilations in the target bulk/para-Ps annihilations and a long exponential tail due to ortho-Ps annihilations in vacuum (with $\sim 142 \mathrm{~ns}$ decay time). Examples of such digitized signals are shown in Fig. 3, inset, where several SSPALS spectra with/without positronium formation are compared: the bare annihilation of positrons on a metallic surface (black curve) and two spectra with Ps obtained using two different reflection targets with two different production efficiencies (gray curves).

AEgIS laser setup for Ps excitation to Rydberg levels is described in detail in Refs. [8] and [14]. It consists of two separate optical setups, fed by the same YAG pump, which generate the two wavelengths required to perform $1 \rightarrow 3$ and $3 \rightarrow 15-23$ transitions, respectively at $205 \mathrm{~nm}$ (UV) and between $1650 \mathrm{~nm}$ and $1720 \mathrm{~nm}$ (mid-IR). Both optical systems use non-linear optical manipulations in optical crystals to generate the required wavelengths from a combination of the first, second and fourth harmonics of a Q-Switched YAG pump. Two mirror optical lines carry the laser pulses to the experimental chamber, where the alignment of the beams is performed 
imaging the beams on a small MACOR screen installed close to the Ps conversion target inside the vacuum. An extra line carries a high-energy $1064 \mathrm{~nm}$ pulse from the YAG pump to perform selective photo-ionization of Ps atoms excited on $n=3$.

\section{Ps laser excitation to Rydberg levels}

Two spectroscopic surveys were conducted to demonstrate the proof-ofprinciple laser excitation chosen for pulsed antihydrogen production (see [15] for further details).

A first survey was conducted sending the $205 \mathrm{~nm}$ UV laser to excite the $1 \rightarrow 3$ transition and an intense $1064 \mathrm{~nm}$ pulse to selectively photoionize the excited atoms only with an efficiency close to $100 \%$. The combined action of the two lasers removed some Ps atoms from the ortho-Ps population, reducing the $142 \mathrm{~ns}$ exponential tail proportionally to the overall laser excitation and ionizaton efficiency. This was observed as a decrease of the Ps signal in the SSPALS spectra (as shown in Fig. 4, left panel). The overall efficiency $S(\%)$ was estimated calculating the relative ratio between the areas of the (averaged and normalized) SSPALS spectra in a selected time window with and without laser ${ }^{1}$. With the laser wavelength set on the transition resonance, $\lambda_{3}=205.047 \mathrm{~nm}$, the excitation and photoionization efficiency was found to be $15.4 \%$. This was mostly due to the limited spectral coverage of the UV laser, $2 \pi \times 48 \mathrm{GHz}$, only partly covering the Doppler-broadened transition. A wavelength scan with the UV laser measuring the excitation efficiency in the window $204.8 \mathrm{~nm} \rightarrow 205.3 \mathrm{~nm}$ allowed to quantify the broadening, found to be about $1 \mathrm{THz}$ (see Fig. 4, right panel). This measurement gave, as a byproduct, an independent estimate of the Ps cloud
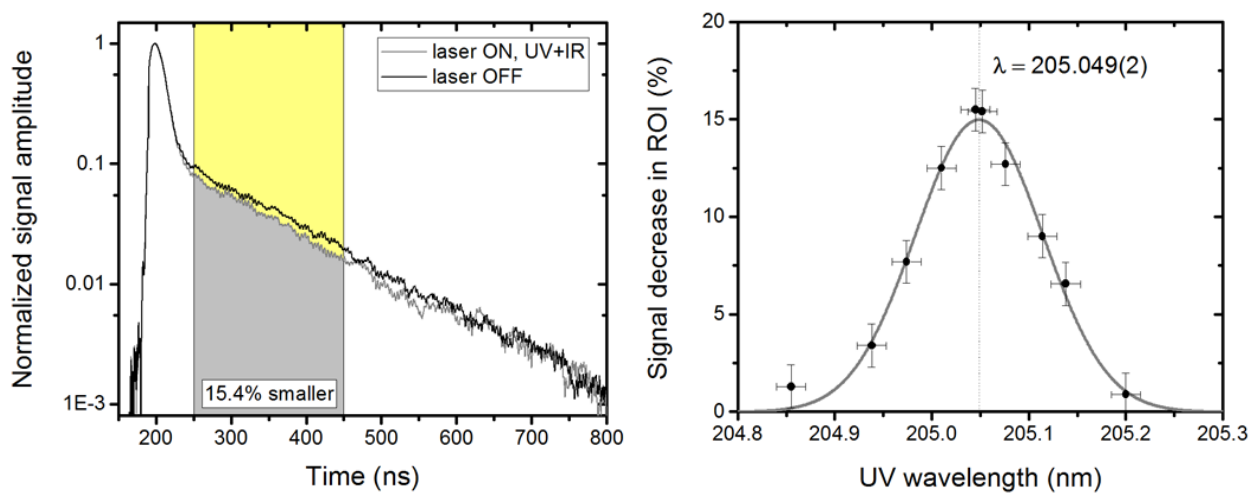

Fig. 4. Spectroscopy of Ps $1 \rightarrow 3$ transition.

\footnotetext{
${ }^{1}$ Further details on the analysis technique are described in [15].
} 
temperature in the direction parallel to the laser, which was found to be $T \sim 1200 \mathrm{~K}$ consistent with time-of-flight measurements performed at room temperature [16].

A second spectroscopic survey was conducted replacing the $1064 \mathrm{~nm}$ photoionization laser with the mid-IR Rydberg excitation laser to scan the wavelength range of 1685-1715 $\mathrm{nm}$ while leaving the UV laser on resonance. Ps atoms were conveyed to long-lived Rydberg levels in the range of 15-18 in place of being ionized, where they survived direct annihilation into $\gamma$ rays for $\gg \mu \mathrm{s}$ and annihilated on the chamber walls. An excess of annihilations was consistently observed in the SSPALS spectra at later times (see Fig. 5, left panel), in agreement with the average flight time of an isotropical $1200 \mathrm{~K}$ source with a Maxwellian distribution of velocities reaching a $5.6 \mathrm{~cm}$ distant obstacle. The amount of signal was quantified in a similar fashion of the first survey, i.e. calculating the relative increment of the SSPALS signal due to the presence of the laser in a selected time window at later times. The scan with the mid-IR laser wavelength (shown in Fig. 5, right panel) showed the multi-peak structure typical of atomic spectra in presence of broadening of the energy levels with an isolated line $(n=15)$ and a quasi-continuum of energy levels $(n>16)$, consistent with the expected broadening caused by the $300 \mathrm{~V} \mathrm{~cm}^{-1}$ electric field present in the experimental chamber (see [17] and [18]). The overall efficiency of the Rydberg excitation could be estimated by the relative reduction in the Ps signal in the same time window used for the photoionization experiment, $7.9 \%$, as in-flight annihilations of high- $n$ states of Ps are negligible. This value, compared to the $15.4 \%$ overall photoionization efficiency, suggests that the sole Rydberg excitation step is somewhat $50 \%$ efficient and can be improved in a future realization of the experiment.
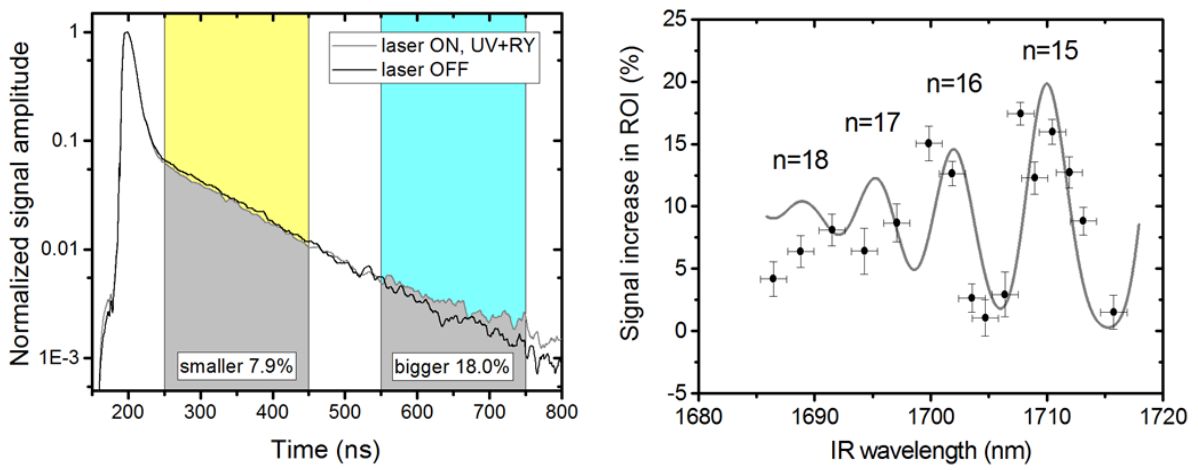

Fig. 5. Spectroscopy of Ps $3 \rightarrow 15-18$ transitions. Left: an example SSPALS spectra with the Ps Rydberg excitation emphasizing the chosen integration windows. Right: the outcome of the IR laser wavelength scan superimposed to the theoretical expectation. 


\section{Ps production from transmission targets}

Antihydrogen production via charge-exchange in AEgIS would greatly benefit from the development of efficient positronium conversion targets in transmission geometry able to operate in a cryogenic environment with the same efficiency and cooling characteristics of present-day reflection targets. With such type of targets, the geometry of the antihydrogen production region (Fig. 1) could be greatly simplified in favour of an axisymmetric geometry with an insertable target, avoiding the extra complexity of off-axis elements.

A recently developed class of transmission targets (see [19]) was tested in the AEgIS dedicated positronium setup to characterize their Ps yield in view of a possible future use in the main experiment (see [20] for further details). These targets were composed by a thin mesoporous film of silica evaporated on a $20 \mathrm{~nm}$-thick carbon foil providing mechanical stability. They were installed on the movable actuator in the center of the experimental chamber, once with the active silica surface facing upstream (reflection configuration), once with the active surface facing downstream (transmission configuration, shown in Fig. 6, left panel).
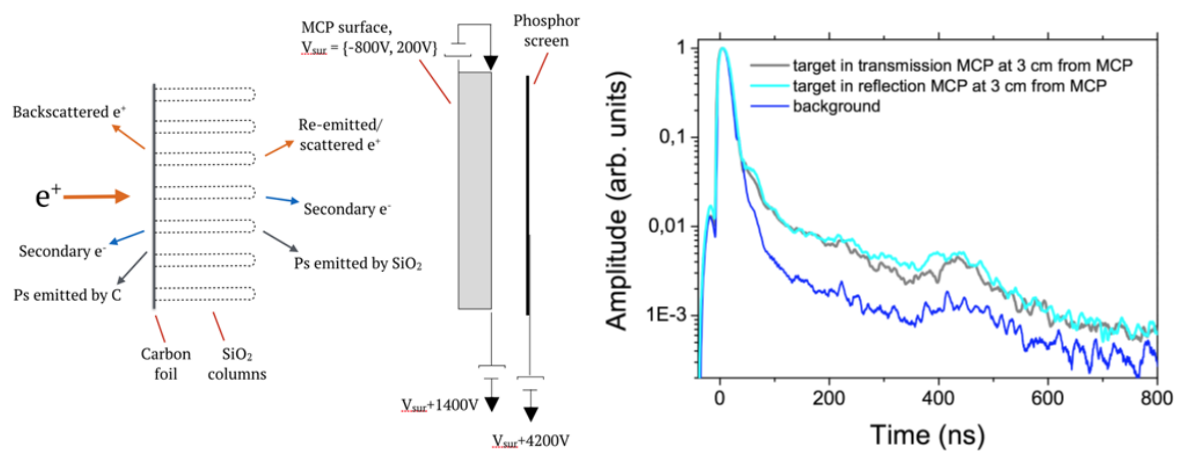

Fig. 6. Ps formation in both transmission and reflection geometries from thin porous silica target evaporated on carbon substrate. Left: layout of the experiment in transmission configuration. Right: SSPALS spectra recorded by the scintillator showing a long tail of Ps formation in both reflection and transmission configurations.

The Ps yield, in both reflection and transmission configurations, was determined from the SSPALS spectra recorded by the external $\mathrm{PbWO}_{4}$ scintillator (see Fig. 6). It was measured integrating the recorded spectra in the Ps signal region (i.e. excluding the implantation peak) and dividing it by the integral of the entire recorded signal (i.e. including the implantation peak). A Ps formation efficiency of $10 \%$ was observed in both reflection and transmission configurations, showing the validity of such a class of targets in 
forming Ps with both orientations. Results obtained are in line with similar results conducted in a different experiment on the same type of targets [21]. These targets, however, are still far from being competitive with the higher yields of reflection targets (for instance, 35\% of AEgIS mesoporous silica reflection targets [7]).

In parallel, the MCP assembly was used to measure forward emitted charged particles from the target. About $10 \%$ of the initial implanted positrons (implanted with $3.3 \mathrm{keV}$ energy) were observed forward re-emitted from the target with an axial energy around $1.2 \mathrm{keV}$. At the same time, the emission of forward secondary electrons of about $1 / 6$ of the initial positrons was reported, with an energy distribution up to $\sim \mathrm{eV}$, in agreement with previous results obtained implanting positron in thin carbon foils [20].

\section{Conclusions}

AEgIS, on its way of first showing pulsed antihydrogen production using resonant charge-exchange between cold antiprotons and Rydberg positronium, has recency demonstrated the feasibility of its in-flight positronium formation and laser excitation scheme to Rydberg levels in a separate setup for spectroscopy experiments. An overall Rydberg excitation efficiency of $8 \%$ has been reported, being limited by the temperature of its Ps source/bandwidth of the present laser setup. AEgIS has been experimenting also novel types of positronium formation targets in transmission geometry for future use, which were found promising besides not being yet competitive with current reflection targets in terms of Ps yield.

\section{REFERENCES}

[1] A. Kellerbauer et al., Nucl. Instrum. Methods Phys. Res. B 266, 351 (2008).

[2] S. Aghion et al., Nat. Commun. 5, 4538 (2014).

[3] C.H. Storry et al., Phys. Rev. Lett. 93, 263401 (2004).

[4] M. Amoretti et al., Nature 419, 456 (2002).

[5] D. Krasnický, R. Caravita, C. Canali, G. Testera, Phys. Rev. A 94, 022714 (2016).

[6] S. Mariazzi, P. Bettotti, R.S. Brusa, Phys. Rev. Lett. 104, 243401 (2010).

[7] S. Aghion et al., Nucl. Instrum. Methods Phys. Res. B 362, 86 (2015).

[8] S. Cialdi et al., Nucl. Instrum. Methods Phys. Res. B 269, 1527 (2011).

[9] A.P. Mills Jr., E.M. Gullikson, Appl. Phys. Lett. 49, 1121 (1986).

[10] J.R. Danielson, D.H.E. Dubin, R.G. Greaves, C.M. Surko, Rev. Mod. Phys. 87, 247 (2015).

[11] C.M. Surko, R.G. Greaves, Phys. Plasmas 11, 2333 (2004). 
[12] D.B. Cassidy, S.H.M. Deng, H.K.M. Tanaka, A.P. Mills Jr., Appl. Phys. Lett. 88, 194105 (2006).

[13] D.B. Cassidy, A.P. Mills Jr., Nucl. Instrum. Methods Phys. Res. A 580, 1338 (2007).

[14] R. Caravita, Laser Apparatus for Exciting Positronium in AEgIS

Positronium Spectroscopy Experiment, Master Thesis, Universitá degli Studi di Milano, 2013.

[15] S. Aghion et al., Phys. Rev. A 94, 012507 (2016).

[16] S. Mariazzi et al., Phys. Rev. B 81, 235418 (2010).

[17] R. Caravita, Towards Measuring Gravity on Neutral Antimatter, Ph.D. Thesis, Universitá degli Studi di Genova, Genova, 2017.

[18] F. Castelli et al., Phys. Rev. A 78, 052512 (2008).

[19] S.L. Andersen et al., Eur. Phys. J. D 68, 124 (2014).

[20] S. Aghion et al., Nucl. Instrum. Methods Phys. Res. B 407, 55 (2017).

[21] S.L. Andersen et al., J. Phys. B 48, 204003 (2015). 